Check for updates

Cite this: RSC Adv., 2019, 9, 17530

Received 5th April 2019

Accepted 16th May 2019

DOI: $10.1039 / c 9 r a 02496 d$

rsc.li/rsc-advances

\section{In vivo evaluation of the subchronic systemic toxicity of akermanite bioceramic for bone regeneration following ISO standard methods}

\author{
Nan Ma, $\dagger^{\text {ac }}$ Bing Ma, $\dagger^{\mathrm{b}}$ Yanling Zhou, ${ }^{\mathrm{b}}$ Haibo Zhu, ${ }^{\mathrm{c}}$ Ying Zhou, ${ }^{\mathrm{c}}$ Zhiguang Huan, ${ }^{\mathrm{b}}$ \\ Peiji Wang ${ }^{\star a}$ and Jiang Chang (DD) *b
}

\begin{abstract}
Although the akermanite $\left(\mathrm{Ca}_{2} \mathrm{MgSi}_{2} \mathrm{O}_{7}\right)$ bioceramic has been confirmed to possess favorable osteogenic activity, until now little was known about its in vivo subchronic systemic toxicity, which is important for determining the biocompatibility and the clinical applications of the material in bone implants. In this study, the subchronic systemic toxicity of akermanite bioceramic was for the first time investigated according to well-accepted ISO standard methods. Following the method, healthy adult Wistar rats were injected with certain amounts of extracts of akermanite bioceramic that was intended to simulate the ionic product during the degradation of the material when implanted into the body. At day 28 after injection, the general body conditions, blood cytology, blood biochemistry and histology of all important organs of the rats were examined. The results showed that there was no significant difference in the hemoglobin concentration, red blood cell count, platelet count and white blood cell count between the rats with injection of akermanite bioceramic extracts and the saline control. The indicators of liver function, including aspartate aminotransferase and alkaline phosphatase, and kidney function, including blood urea nitrogen and creatinine, did not show significant difference between the two groups $(P>$ 0.05). In addition, the results of histological examination showed that the extract of akermanite bioceramic did not cause any pathological changes to important organs such as the heart, liver and kidneys. These findings demonstrated that the ionic product derived from the degradation of akermanite bioceramic did not cause in vivo subchronic systemic toxicity. The results of the current study provided more strengthened evidence for the biosafety of akermanite bioceramic and suggest that this material with desirable biocompatibility may be a potential candidate for orthopedic clinical applications.
\end{abstract}

\section{Introduction}

Although autologous and allogeneic bones are considered as good choices for bone repair, their source is limited and transplantation of these bones may cause serious shortcomings such as infections and immune rejection. ${ }^{1-5}$ Therefore, some synthetic materials are employed for bone repair in clinical practice. Calcium phosphate bioceramics are similar to natural bone tissues in the inorganic composition (hydroxyapatite) and have favorable biocompatibility, making them widely used for bone repair in clinical practice. The osteogenic activity of sintered calcium phosphate

${ }^{a}$ Department of Hand Surgery, Second Affiliated Hospital of Suzhou University, Suzhou 215000, China

${ }^{b}$ State Key Laboratory of High Performance Ceramics and Superfine Microstructure, Shanghai Institute of Ceramics, Chinese Academy of Sciences, 1295 Dingxi Road, Shanghai 200050, China. E-mail: jchang@mail.sic.ac.cn

${ }^{c}$ Department of Orthopaedics, Xuhui Central Hospital of Shanghai, Shanghai 200031, China

$\dagger$ The two authors contributed to the work equally. bioceramics still needs improvement, and in some case the degradability of the material is poor. ${ }^{6-10}$ Therefore, investigation of degradable bioceramics with favorable osteogenic activity has always been a focus in the field of biomaterials for bone repair.

It is well known that silicon is an important trace element for bone regeneration and repair, which can up-regulate the expression of osteogenesis-related genes, activate the osteogenesis related signaling pathways, and promote the osteogenic differentiation of bone marrow mesenchymal stem cells. ${ }^{11-14}$ There has been great progress in the development of silicate-based bioactive ceramics in the past decade. Many studies have confirmed that these ceramics can release $\mathrm{Si}$ ions to significantly promote the proliferation and differentiation of bone cells. ${ }^{15-17}$ Compared with calcium phosphate ceramics, silicate based bioceramics have significantly better osteogenic activity and in vivo degradability, making them have great potential for more efficient bone repair. ${ }^{18-23}$

Akermanite $\left(\mathrm{Ca}_{2} \mathrm{MgSi}_{2} \mathrm{O}_{7}\right)$ is a typical type of silicate based bioceramics, which was capable releasing calcium, magnesium and silicate ions in physiological environment, and 
some previous studies have demonstrated that implantation of porous akermanite in the injured femur of rabbits could promote the bone regeneration and angiogenesis as compared to $\beta$-TCP treated rabbits. ${ }^{24-26}$ These results suggested that akermanite bioceramic could consider as a good candidate for bone repair with good bioactivity. However, it should be noted that these studies have been focused on the effects of the material on the osteogenic differentiation and bone regeneration, whereas little is known about the subchronic systemic toxicity of degraded products of these material, which is crucial for its future clinical application. Different from the test on bone-forming ability of an implant, the subchronic systemic toxicity test is intended to evaluate the possible effects of the absorption, distribution, or metabolism of products originating from the material, involving parts of the body or organs not in direct contact with the material. Considering the fact that akermanite bioceramic is biodegradable and its ionic biodegradation products, including $\mathrm{Ca}, \mathrm{Mg}$ and $\mathrm{Si}$ ions, are likely to be involved in circulation, concerns may rise over its influence on blood and organ function. It has been reported that silicate or magnesium at a high concentration may inhibit the cell proliferation and even induce cell apoptosis, and overloading of these ionsmay cause damage on lung, cardiovascular system, liver, kidney and other organs. ${ }^{27-33}$ Thus, although akermanite has favorable osteogenicity, the distribution and subchonic influence of degraded products of akermanite are still poorly understood, and more in-depth studies are needed to confirm its biological safety.

This study therefore aimed to investigate the in vivo subchronic systemic toxicity of akermanite according to the methods that are recommended in ISO 10993-11:2017 and ISO 10993-12:2012. The extracts of akermanite was injected into rats by peritoneal injection daily, and blood cytology, blood biochemistry and histology of different organs were examined over 28 days. Our findings may provide guidance on the subchronic systemic toxicity and biosafty on akermanite based bioceramic, and together with the results that have demonstrate the in vivo bone forming ability of the material, the outcome of the present study may provide a wide scope of view on the safety of akermanite bioceramic for its potential application in bone repair and regeneration.

\section{Materials and methods}

\subsection{Materials}

Akermanite powders with the powder size between 25 and 40 $\mu \mathrm{m}$ were purchased from Huaqiao New Materials Company, China.

Healthy adult Wistar rats were provided by the Experimental Animal Center of the Sixth People's Hospital in Shanghai. All animal procedures were performed in accordance with the Guidelines for Care and Use of Laboratory Animals of Shanghai Jiao Tong University (Shanghai, China) and approved by the Animal Ethics Committee of Shanghai Jiao Tong University School of Medicine (Shanghai, China).

\subsection{Preparation of akermanite extracts}

Extracts of akermanite were prepared following the method described in 10.3 of the standard ISO 10993-12:2012 (Biological evaluation of medical devices - Part 12: Sample preparation and reference materials). Briefly, akermanite powders were soaked in normal saline at a ratio of $0.2 \mathrm{~g} \mathrm{~mL}^{-1}$ at $121^{\circ} \mathrm{C}$ for $1 \mathrm{~h}$. Then the supernatant was collected and sterilized by filtration through a filter ( $0.22 \mu \mathrm{m}$, Millipore).

\subsection{Injection of akermanite extracts into rats}

The injection of akermanite extracts was performed according to the method described in 3.8 of the standard ISO 1099311:2017 (Biological evaluation of medical devices - Part 11: Tests for systemic toxicity). Healthy adult Wistar rats weighing from 180 to $220 \mathrm{~g}(n=40)$ (male: $n=20$; female: $n=20$ ) were randomly divided into 2 groups: akermanite group (AKE; male: $n=10$; female: $n=10$ ) and control group (male: $n=10$; female: $n=10$ ). In AKE group, the akermanite extracts were injected at an extracts volume/rat weight ratio of $20 \mathrm{~mL} \mathrm{~kg}^{-1}$ by peritoneal injection once a day for 28 days. In control group, normal saline was injected at the same conditions. After each injection, the general conditions (survival, food/water intake and body weight) and toxic reactions were monitored. All the animals were weighed once every 3 days.

\subsection{Examination of blood cytology and biochemistry}

At the end of injection, blood was collected from the heart and processed for cytological and biochemical examinations (reagents were purchased from MaiRui Medical Co., Ltd). Following parameters were detected: hemoglobin concentration (HCG), red blood cell count (RBC), platelet count (PLT), white blood cell (WBC) count, WBC subsets, hematocrit, aspartate aminotransferase (AST), alanine aminotransferase (ALT), total protein (TP), albumin (ALB), ratio of albumin to globulin (A/G), globulin (GLB), AKP, blood urea nitrogen (BUN), creatinine (CRE), urea (UREA), blood glucose (GLU), total cholesterol (CHOL), triglyceride (TG), potassium (K), sodium $(\mathrm{Na})$, chlorine $(\mathrm{Cl})$ and calcium $(\mathrm{Ca})$.

\subsection{Autopsy and histological examination}

At the end of injection, animals were sacrificed, followed by autopsy. The main organs were weighed (brain, lungs, heart, liver, spleen, stomach, kidneys and gonads), and the pathological changes were recorded. Then, these organs were fixed in $10 \%$ formalin, embedded in paraffin and sectioned for $\mathrm{HE}$ staining. Sections were observed under a light microscope. In addition, the ratio of organ weight to body weight was calculated.

Sections were prepared as above mentioned, and the ion distribution was observed by scanning electron microscope (SEM, SU8220, HITACHI, Japan) equipped with an energy dispersive spectrometer (EDS). 


\subsection{Statistical analysis}

Data are expressed as mean \pm standard deviation (SD) and compared with $t$ test between groups. Credibility of $95 \%$ was employed to determine the statistical significance.

\section{Results}

\subsection{The properties of akermanite extracts}

The akermanite particles and the extracts of akermanite are shown in Fig. 1A and B, and it can be seen that the particles were white and the akermanite extracts were colorless and transparent. The ion concentrations of akermanite extracts and normal saline were shown in Table 1 . The concentrations of $\mathrm{Ca}$, $\mathrm{Mg}$ and $\mathrm{Si}$ ions of akermanite extracts were 60.20, 0.52 and $32.05 \mathrm{ppm}$, respectively, which were much higher than those of normal saline.

\subsection{General observation and body weight of animals}

All the rats survived during the test, and no obvious toxic reactions were observed during the overall study period. The body weight was measured once every 3 days, and the results were displayed in Fig. 2. The body weight increased gradually in control group. In AK group, there was no loss of appetite, and the body weight also increased over time. There was no significant difference in the body weight between groups $(P>0.05)$.

\subsection{Blood cytology assay}

At the end of study, rats were sacrificed and the blood was collected from the heart for cytological examination. It can be seen that there were no obvious reductions in the HCG, RBC, PLT, WBC, WBC subsets and hematocrit after injection. In addition, the indexes of HCG, RBC, PLT, WBC, WBC subsets and hematocrit did show significant difference between the two groups $(P>0.05)$ (Table 2$)$.

\subsection{Serum biochemistry assay}

At the end of study, rats were sacrificed and the blood was collected from the heart for biochemical examination. There were no significant increases in the AST, ALT, AKP, TP, ALB, A/ G, GLB, BUN, CRE, UREA, blood lipids, GLU, K, Na, Cl and Ca.
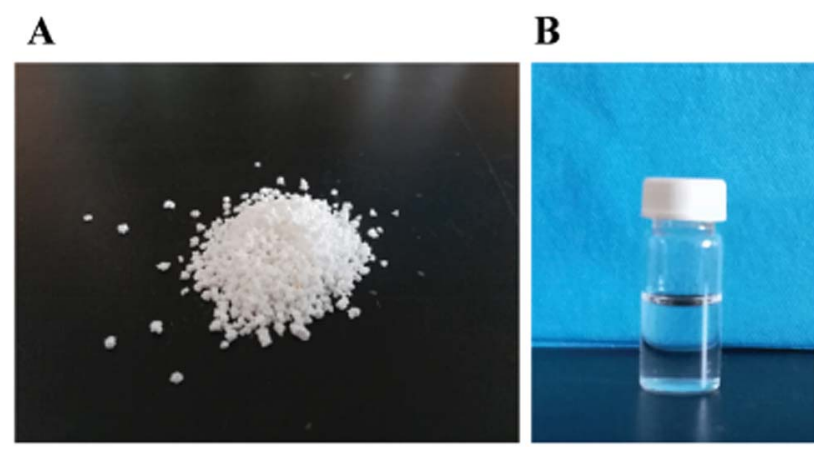

Fig. 1 The particles of akermanite (A) and the extracts of akermanite (B).
Table 1 Ion concentrations of akermanite and normal saline (ppm)

\begin{tabular}{lclr}
\hline & $\mathrm{Ca}$ & $\mathrm{Mg}$ & \multicolumn{1}{c}{$\mathrm{Si}$} \\
\hline Akermanite extracts & 60.2 & 0.52 & 32.05 \\
Normal saline & 0.28 & 0.022 & 0.13
\end{tabular}

In addition, significant differences in these parameters were not seen between groups $(P>0.05)$ (Table 3$)$.

\subsection{Organ to body weight ratios}

After 28 days, rats were sacrificed, and the brain, lungs, heart, liver, spleen, stomach, kidneys and gonads were harvested for pathological examination. Macroscopic examination showed no congestion, hemorrhage, edema and necrosis. Each organ was weighed and the organ to body weight ratio was calculated. As shown in Table 4, there was no significant difference in the organ to body weight ratio between two groups $(P>0.05)$.

\subsection{Pathological examinations}

The main organs of the rats, including liver, renal and heart, were collected after their sacrifice and fixed in $10 \%$ formalin, and then were processed for HE staining. The results of pathological examinations of the organs were presented in Fig. 3. In AKE group and control group, pathological changes were not observed in all main organs such as liver, renal, and heart. In both groups, liver capsule was complete, the hepatic lobules displayed normal structure, hepatocytes had normal morphology, and there were no aggregation of inflammatory cells and cell necrosis (Fig. 3A and B). The renal capsule was complete, and the structure of renal cortex and medulla was normal (Fig. 3C and D). The heart structure was complete, and cardiomyocytes displayed continuous and regular arrangement. The nucleus was clear and identifiable, and there were no cell congestion, edema and necrosis (Fig. $3 \mathrm{E}$ and F).

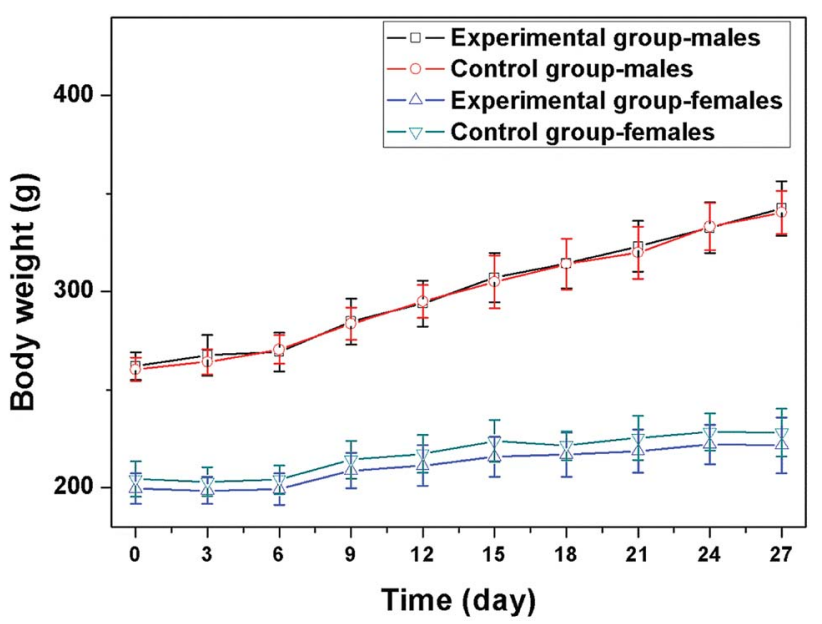

Fig. 2 The body weight of the two groups of rats at different time points among 4 weeks. 
Table 2 Blood cytology of rats in two groups $(X \pm s, n=10)$

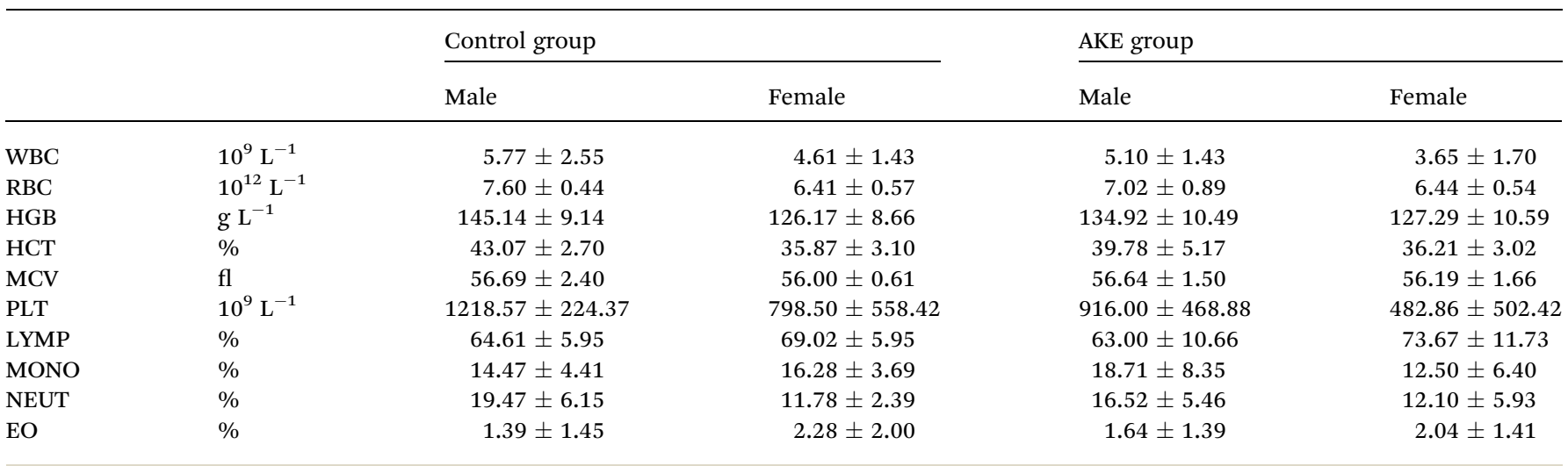

The elemental distribution of $\mathrm{Ca}, \mathrm{Mg}$ and $\mathrm{Si}$ in the organs of rats in AKE group and control group are shown in Fig. 4. At the end of the study (28 days), $\mathrm{Ca}, \mathrm{Mg}$ and $\mathrm{Si}$ were not found in the heart, liver and kidney of rats in both groups.

\section{Discussion}

The implants that are to be used for bone repair should have favorable biocompatibility and safety. In this sense, the metabolic products of an implanted biodegradable biomaterial should be safely excreted from the human body and should not be accumulated in organs and tissues, which is important to the avoidance of the toxic reactions of the body to implants.

A variety of studies have shown that akermanite bioceramics can release some ions (such as $\mathrm{Si}$ and $\mathrm{Mg}$ ) to promote the bone regeneration during its degradation in vivo. ${ }^{24,25}$ However, until now little is known about the subchronic systemic toxicity of akermanite bioceramics, including vital signs, blood cytology, serum biochemistry, pathological examination of organs, making the biocompatibility of the material still not well illuminated. ISO 10993-11:2017 is a well-established standard with authority that provides the instruction for the evaluation of subchronic systemic toxicity of medical devices, including bone implants. Therefore, in this study, for the first time the subchronic systemic toxicity of akermanite bioceramics according to ISO 10993-11:2017 was systemically investigated in rats, which may provide evidence on the biological safety of akermanite bioceramic.

In general, all the rats survived throughout the study, and of specially interest, the ionic degradation product of akermanite bioceramic did not cause any significant adverse effect on the main cellular components of blood. According to previous studies, it has been reported that overloading of $\mathrm{Si}$ and $\mathrm{Mg}$ in blood at a high concentration may cause damage to RBC, WBC, PLT and HCG and inhibit the proliferation of these cells, resulting in the reduction in $\mathrm{RBC}$, WBC, WBC subsets, including hematocrit and HCG, which may lead to tissue hypoxia, low immune status and functional disorder of body. ${ }^{27,28}$ In present study, it was found that the extract of akermanite bioceramics did not cause any significant influence on the features of RBC, WBC, PLT and HCG, as there were no remarkable difference between the groups. These findings

Table 3 Blood biochemistry of rats in two groups $(X \pm s, n=10)$

\begin{tabular}{|c|c|c|c|c|c|}
\hline & & \multicolumn{2}{|l|}{ Control group } & \multicolumn{2}{|l|}{ AKE group } \\
\hline & & Male & Female & Male & Female \\
\hline $\mathrm{AST}$ & $\mathrm{U} \mathrm{L}^{-1}$ & $222.5 \pm 99.3$ & $211.3 \pm 148.7$ & $183.0 \pm 55.7$ & $160.9 \pm 39.2$ \\
\hline ALT & $\mathrm{U} \mathrm{L}^{-1}$ & $48.9 \pm 9.4$ & $73.5 \pm 78.4$ & $53.2 \pm 17.8$ & $43.0 \pm 14.8$ \\
\hline $\mathrm{TP}$ & $g \mathrm{~L}^{-1}$ & $62.9 \pm 5.3$ & $67.3 \pm 4.9$ & $62.1 \pm 3.8$ & $66.7 \pm 4.2$ \\
\hline ALB & $\mathrm{g} \mathrm{L}^{-1}$ & $37.9 \pm 2.6$ & $40.4 \pm 4.1$ & $38.8 \pm 2.7$ & $41.2 \pm 2.4$ \\
\hline $\mathrm{A} / \mathrm{G}$ & $\%$ & $1.5 \pm 0.2$ & $1.5 \pm 0.2$ & $1.7 \pm 0.1$ & $1.6 \pm 0.2$ \\
\hline GLOB & $\mathrm{g} \mathrm{L}^{-1}$ & $25.0 \pm 3.9$ & $26.9 \pm 1.9$ & $23.3 \pm 1.7$ & $25.5 \pm 3.3$ \\
\hline AKP & $\mathrm{U} \mathrm{L}^{-1}$ & $263.1 \pm 66.2$ & $181.4 \pm 70.3$ & $280.8 \pm 63.7$ & $154.3 \pm 47.5$ \\
\hline BUN & $\mathrm{mmol} \mathrm{L}^{-1}$ & $6.3 \pm 0.6$ & $5.5 \pm 0.8$ & $6.8 \pm 0.5$ & $6.2 \pm 0.9$ \\
\hline CREA & $\mathrm{mmol} \mathrm{L}^{-1}$ & $15.8 \pm 7.4$ & $23.1 \pm 3.8$ & $14.5 \pm 9.2$ & $21.9 \pm 2.2$ \\
\hline URCA & $\mathrm{mmol} \mathrm{L}^{-1}$ & $325.4 \pm 108.4$ & $248.5 \pm 75.4$ & $303.5 \pm 96.6$ & $251.9 \pm 140.9$ \\
\hline GLU & $\mathrm{mmol} \mathrm{L}^{-1}$ & $13.5 \pm 4.5$ & $8.7 \pm 2.0$ & $13.1 \pm 4.3$ & $10.9 \pm 5.9$ \\
\hline CHOL & $\mathrm{mmol} \mathrm{L}^{-1}$ & $2.2 \pm 0.3$ & $2.2 \pm 0.4$ & $1.9 \pm 0.2$ & $2.1 \pm 0.2$ \\
\hline TRIG & $\mathrm{mmol} \mathrm{L}^{-1}$ & $1.4 \pm 0.3$ & $0.8 \pm 0.1$ & $1.8 \pm 0.8$ & $0.9 \pm 0.3$ \\
\hline $\mathrm{K}$ & $\mathrm{mmol} \mathrm{L}^{-1}$ & $9.6 \pm 1.9$ & $7.9 \pm 1.6$ & $9.7 \pm 3.3$ & $7.7 \pm 1.8$ \\
\hline NA & $\mathrm{mmol} \mathrm{L}^{-1}$ & $142.2 \pm 2.2$ & $141.9 \pm 1.6$ & $143.2 \pm 3.5$ & $143.3 \pm 3.0$ \\
\hline $\mathrm{CL}$ & $\mathrm{mmol} \mathrm{L}^{-1}$ & $100.8 \pm 2.1$ & $101.3 \pm 1.3$ & $101.7 \pm 2.1$ & $103.3 \pm 1.6$ \\
\hline
\end{tabular}


Table 4 Ratio of organ weight to body weight in two groups $(X \pm s, n=10)$

\begin{tabular}{|c|c|c|c|c|c|}
\hline & & \multicolumn{2}{|l|}{ Control group } & \multicolumn{2}{|l|}{ AKE group } \\
\hline & & Male & Female & Male & Female \\
\hline Spleen/body & $\%$ & $0.28 \pm 0.029$ & $0.31 \pm 0.021$ & $0.32 \pm 0.028$ & $0.33 \pm 0.026$ \\
\hline Kidney/body & $\%$ & $0.65 \pm 0.036$ & $0.72 \pm 0.051$ & $0.68 \pm 0.041$ & $0.73 \pm 0.032$ \\
\hline Gonad/body & $\%$ & $0.95 \pm 0.002$ & $0.036 \pm 0.0081$ & $0.96 \pm 0.021$ & $0.039 \pm 0.016$ \\
\hline Lung/body & $\%$ & $0.42 \pm 0.28$ & $0.52 \pm 0.18$ & $0.46 \pm 0.21$ & $0.56 \pm 0.13$ \\
\hline Stomach/body & $\%$ & $0.53 \pm 0.11$ & $0.56 \pm 0.28$ & $0.59 \pm 0.21$ & $0.58 \pm 0.31$ \\
\hline
\end{tabular}
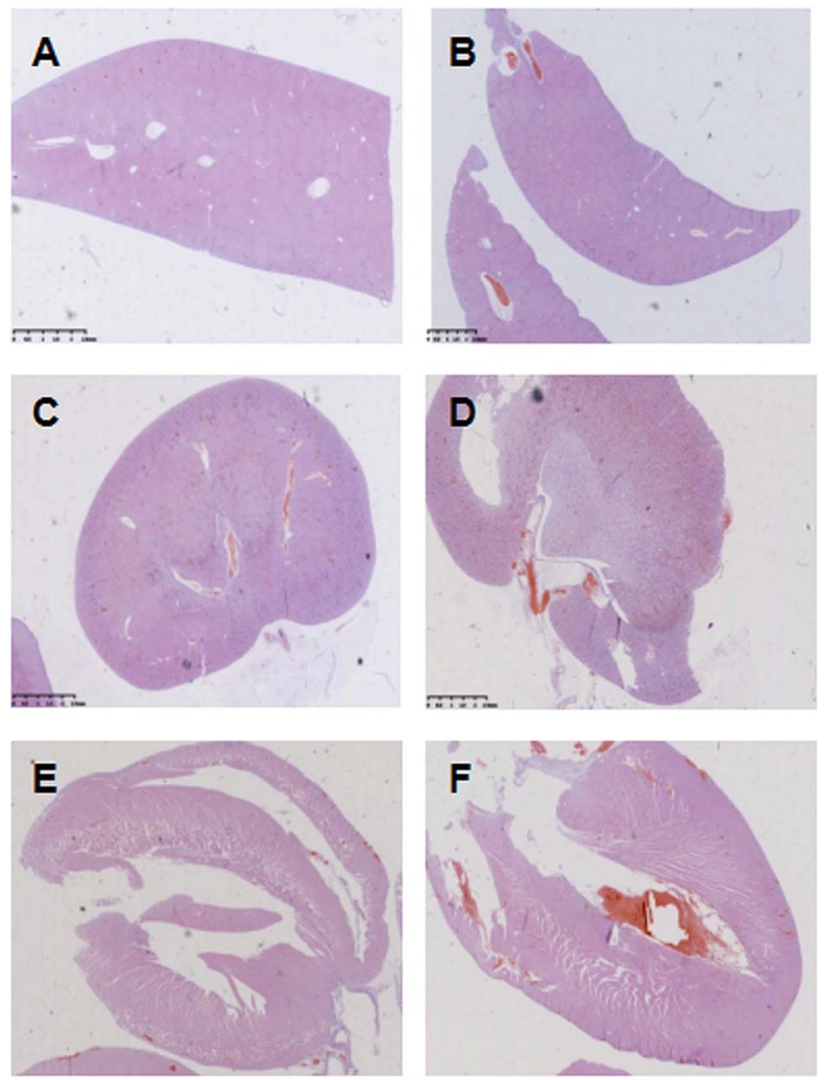

Fig. 3 Pathological result of liver ( $A$ and $B)$, renal $(C$ and $D)$ and heart $(E$ and $F$ ) of the control group (,$C$ and $E$ ) and the AKE group (B, D and F) of rats as observed by $\mathrm{HE}$ staining.

indicate that the $\mathrm{Si}$ and $\mathrm{Mg}$ released by akermanite bioceramics did not affect the metabolism of cells in the blood, indicating that the degradation of the material would not cause significant toxicity to blood cells. It is reasonable to believe that the good compatibility of akermanite bioceramic could be attributed to the fact that the ionic concentration of its degradation product was relatively low, which was well tolerated by the blood cells.

In some previous studies it has been demonstrated that $\mathrm{Si}$ and $\mathrm{Mg}$ in daily intake can be effectively metabolized in the liver and expelled through the stool and urine. However, overdose $\mathrm{Si}$ or $\mathrm{Mg}$ through circulation may cause damage to the liver and kidney. ${ }^{34,35}$ Namely, it may cause hepatocyte necrosis and rupture, leading to the release of AST, ALT and ALP into the blood, which cause increases in blood enzymes. In the present study, it was found that the injection of the extract of akermanite bioceramic by peritoneal injection did not cause significant increases in the ALT, AST, ALP, urea and serum creatinine (parameters related to kidney function), suggesting that the $\mathrm{Si}$ and $\mathrm{Mg}$ released from akermanite bioceramic were well below the toxic dose, and thus can be safely and effectively metabolized in vivo and would not cause toxicity to the liver and kidney.

Once a biodegradable bone graft is implanted, in common sense there will be concern raising over the potential accumulation of its degradation product in organs, which may have toxic effects. In the present study, important organs including the heart, live and kidney were both macroscopically and microscopically examined after injection of the extract of akermanite bioceramics, and pathological phenomenon such as congestion, hemorrhage, hyperplasia, edema and necrosis were not observed in these organs. These results suggest that ionic degradation products did not cause damage to important organs. In addition, $\mathrm{Si}$ and $\mathrm{Mg}$ were not detectable in liver and kidney after 28 days of continuous injection of the extract of akermanite bioceramic. Considering the fact that liver and kidney are known to be responsible for the metabolism of $\mathrm{Si}$ and $\mathrm{Mg}$, it is reasonable to believe that the metabolic products of akermanite are normally metabolized without either accumulating or causing damage to these organs.

Taken together, the results of the present study demonstrated that the ionic degradation products of akermanite bioceramic did not cause any adverse effect on structure and function of the blood cells and all important organs, and were safely metabolized by the body. According to the instruction of the ISO standard related to the evaluation of the subchronic systemic toxicity of an implant, we believe that the results may provide strengthened evidence that akermanite bioceramics possess good biosafety in terms of subchronic systemic toxicity. The results of the present study, together with those of previous studies that have demonstrated the outstanding osteogenetic bioactivity of akermanite bioceramic, would further confirm that the material would be a bioactive bone graft material with desirable biosafety for orthopedic applications. 

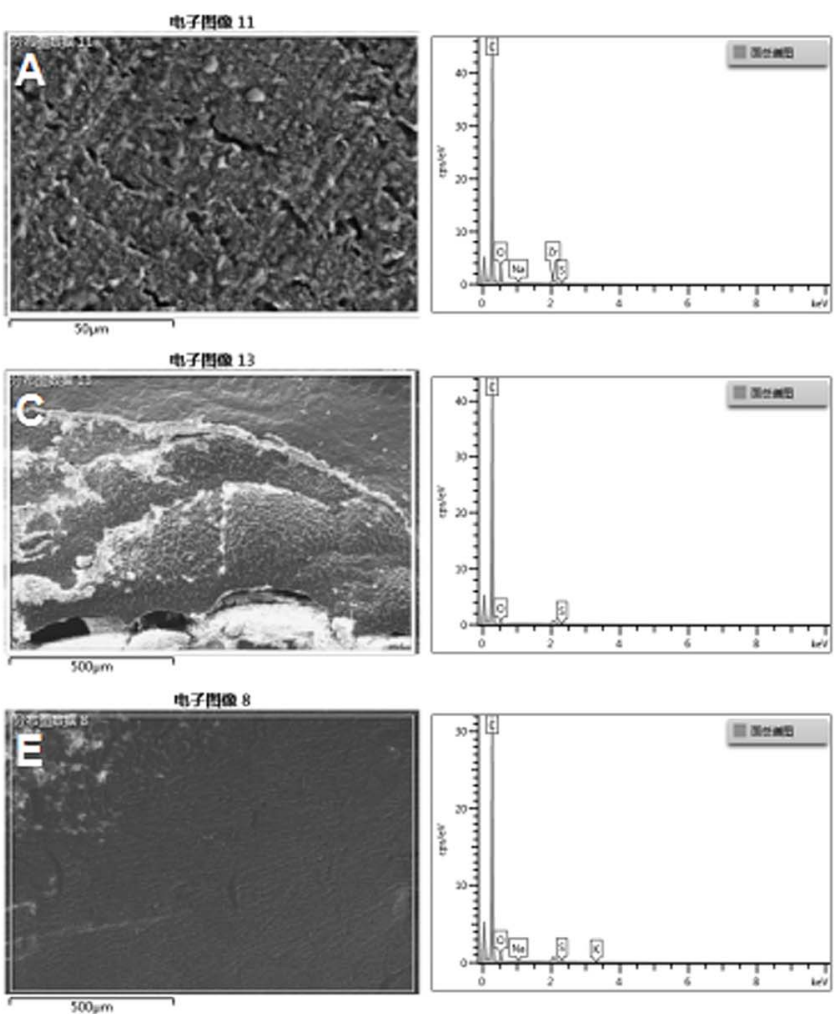
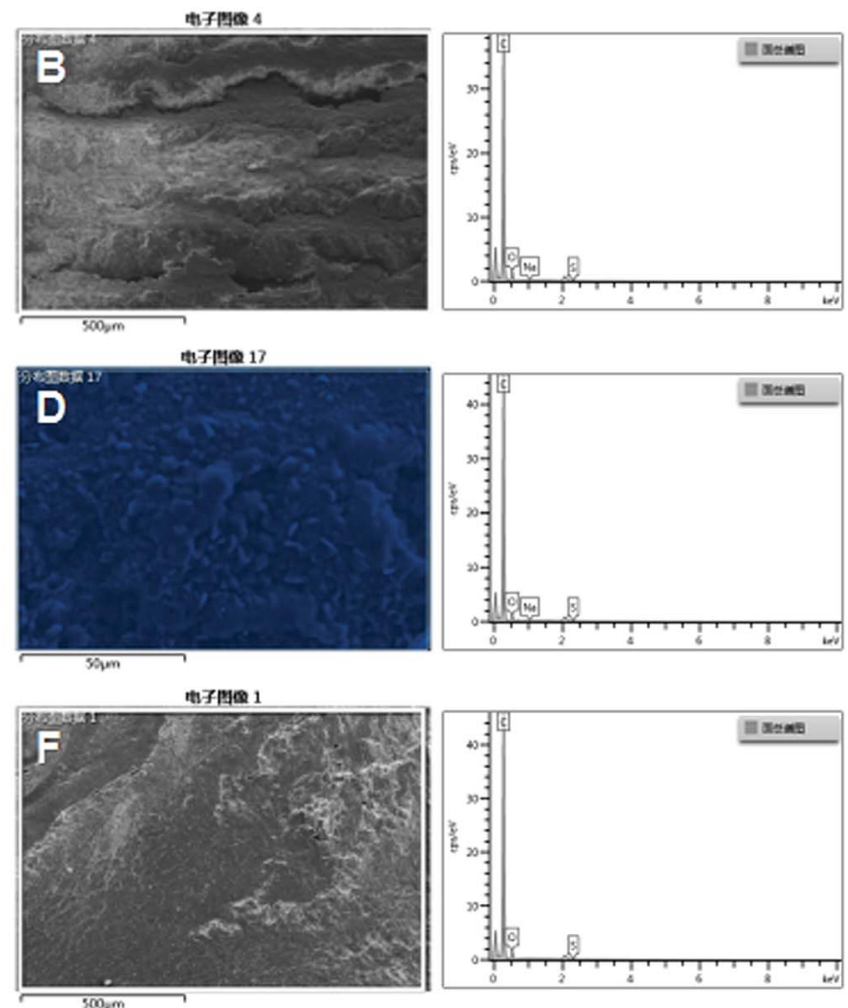

Fig. 4 The elemental concentrations of $\mathrm{Ca}, \mathrm{Mg}$ and $\mathrm{Si}$ in the organs of rats including liver, renal, and heart of the control group (A, C, and $\mathrm{E}$ ) and the AKE group ( $B, D$, and $F$ ).

\section{Conclusions}

In the present study, the subchronic systemic toxicity of the ionic degradation products of akermanite bioceramic was investigated according to the instruction of ISO standard. It was found that the injection of the ionic extract of akermanite bioceramics did not induce any significant adverse effect on the numbers and function of main blood cells. In addition, no pathological change was observed in all important organs of the animals after injection of the extracts, and the functions of the liver and kidney as the main metabolizing organs were not affected. These results demonstrated that akermanite bioceramic did not have any subchronic systemic toxicity, and considering its well-accepted osteogenic bioactivity, showed great potential as bioactive bone graft materials for orthopedic applications.

\section{Conflicts of interest}

There are no conflicts to declare.

\section{Acknowledgements}

The authors wish to express their thanks to National Key R\&D Program of China (No. 2018YFB1105600), the National Natural Science Foundation of China (No. 81671830), Medical Project of Suzhou (No. Szxk201802), and Science Development Plan of Suzhou (No: SZS201720) for financial support.

\section{References}

1 J. Breeze, J. Patel, M. S. Dover and R. W. Williams, Br. J. Oral Maxillofac. Surg., 2017, 55, 830-833.

2 M. K. Sen and T. Miclau, Injury, 2007, 38, S75-S80.

3 A. Sakkas, F. Wilde, M. Heufelder, K. Winter and A. Schramm, Int. J. Implant Dent., 2017, 3, 23.

4 A. Sakkas, A. Schramm, K. Winter and F. Wilde, Risk factors for post-operative complications after procedures for autologous bone augmentation from different donor sites, J. Cranio Maxill. Surg., 2018, 46, 312-322.

5 R. Windhager, G. M. Hobusch and M. Matzner, Orthopade, 2017, 46, 656-664.

6 S. V. Dorozhkin, Biomatter, 2011, 1(1), 3-56.

7 H. P. Stallmann, C. Faber, A. L. Bronckers, A. V. Nieuw Amerongen and P. I. Wuisman, J. Antimicrob. Chemother., 2004, 54, 472-476.

8 A. Matsumine, K. Takegami, K. Asanuma, T. Matsubara, Y. Nakamura, A. Uchida and A. Sudo, Int. J. Clin. Oncol., 2011, 16, 101-108.

9 K. Na, S. W. Kim, B. K. Sun, B. G. Woo, H. N. Yang, H. M. Chung and K. H. Park, Biomaterials, 2007, 28, 26312637.

10 Y. Mizushima, T. Ikoma, J. Tanaka, K. Hoshi, T. Ishihara, Y. Ogawa and A. Ueno, J. Controlled Release, 2006, 110, 260-265. 
11 D. Correa, A. Almirall, R. García-Carrodeguas, L. A. dos Santos, A. H. De Aza, J. Parra and J. A. Delgado, J. Biomed. Mater. Res., Part A, 2014, 102, 3693-3703.

12 X. Y. Yang, M. Liu, Y. Zhao, H. Y. Jia, S. Z. Xu, X. G. Li, X. Y. Chen, F. Zhang, C. Y. Gao and Z. R. Gou, J. Mater. Chem. B, 2014, 2, 3830-3838.

13 A. A. Eid, K. A. Hussein, L. N. Niu, G. H. Li, I. Watanabe, M. Al-Shabrawey, D. H. Pashley and F. R. Tay, Acta Biomater., 2014, 10, 3327-3334.

14 J. Lu, J. Wei, Y. Yan, H. Li, J. Jia, S. Wei, H. Guo, T. Xiao and C. Liu, J. Mater. Sci.: Mater. Med., 2011, 22, 607-615.

15 K. L. Lin, L. G. Xia, H. Y. Li, H. B. Pan, Y. Xu, W. W. Lu, Z. Zhang and J. Chang, Biomaterials, 2013, 34, 10028-10042.

16 C. Wang, K. L. Lin and J. Chang, Biomaterials, 2013, 34, 6477.

17 S. F. Xu, K. L. Lin, Z. Wang, J. Chang, L. Wang, J. X. Lu and C. Q. Ning, Biomaterials, 2008, 29, 2588-2596.

18 C. T. Wu and J. Chang, J. Biomater. Appl., 2006, 21, 119-129.

19 C. T. Wu and J. Chang, J. Biomed. Mater. Res., Part B, 2007, 83, 153-160.

20 H. L. Sun, C. T. Wu, K. R. Dai, J. Chang and T. T. Tang, Biomaterials, 2006, 27, 5651-5657.

21 Q. H. Liu, L. Cen, S. Yin, L. Chen, G. P. Liu, J. Chang and L. Cui, Biomaterials, 2008, 29, 4792-4799.

22 Y. Huang, X. G. Jin, X. L. Zhang, H. L. Sun, J. W. Tu, T. T. Tang, J. Chang and K. R. Dai, Biomaterials, 2009, 30, 5041-5048.
23 M. Diba, O. M. Goudouri, F. Tapia and A. R. Boccaccini, Curr. Opin. Solid State Mater. Sci., 2014, 18, 147-167.

24 C. T. Wu, J. Chang, S. Y. Ni and J. Y. Wang, J. Biomed. Mater. Res., Part A, 2006, 76, 73-80.

25 W. Y. Zhai, H. X. Lu, L. Chen, X. T. Lin, Y. Huang, K. R. Dai, K. Naoki, G. P. Chen and J. Chang, Acta Biomater., 2012, 8, 341-349.

26 Y. Huang, X. G. Jin, X. L. Zhang, H. L. Sun, J. W. Tu, T. T. Tang, J. Chang and K. R. Dai, Biomaterials, 2009, 30, 5041-5048.

27 M. Y. Shie, S. J. Ding and H. C. Chang, Acta Biomater., 2011, 7, 2604-2614.

28 T. Yu, D. Hubbard, A. Ray and H. Ghandehari, J. Controlled Release, 2011, 163, 46-54.

29 R. Kumar, I. Roy, T. Y. Ohulchanskky, L. A. Vathy, E. J. Bergey, M. Sajjad and P. N. Prasad, ACS Nano, 2010, 4, 699-708.

30 H. J. Eom and J. Choi, Toxicol. In Vitro, 2009, 23, 1326-1332. 31 J. S. Kim, T. J. Yoon, K. N. Yu, B. G. Kim, S. J. Park, H. W. Kim, K. H. Lee, S. B. Park, J. K. Lee and M. H. Cho, Toxicol. Sci., 2005, 89, 338-347.

32 M. Amin, E. M. Ewais, Y. M. Ahmed, E. A. Ashor, U. Hess and K. Rezwan, Bioceram. Dev. Appl., 2016, 6(1), 088.

33 E. M. Ewais, A. Moustafa, K. Pardun and K. Rezwan, J. Mater. Sci., 2015, 5, 21-36.

34 A. Nemmar, P. Yuvaraju, S. Beegam, J. Yasin, E. E. Kazzam and B. H. Ali, Int. J. Nanomed., 2016, 11, 919-927.

35 R. Swaminathan, Clin. Biochem. Rev., 2003, 24, 47-66. 